\title{
Philosophiques
}

\section{Le rapport masse-élite comme modèle canonique de la dialectique sociale}

\section{Christiane Gohier}

Volume 11, numéro 2, octobre 1984

Égalité, justice et différence

URI : https://id.erudit.org/iderudit/203261ar

DOI : https://doi.org/10.7202/203261ar

Aller au sommaire du numéro

Éditeur(s)

Société de philosophie du Québec

ISSN

0316-2923 (imprimé)

1492-1391 (numérique)

Découvrir la revue

Citer cet article

Gohier, C. (1984). Le rapport masse-élite comme modèle canonique de la dialectique sociale. Philosophiques, 11(2), 337-348.

https://doi.org/10.7202/203261ar
Résumé de l'article

L'étude des rapports sociaux entre les hommes permet de dégager un invariant structurel des sociétés avec accumulation : le fait de la domination économico-socio-politique d'un petit nombre parmi les hommes sur le plus grand nombre. Qu'il s'agisse du couple maître-esclave dans la société grecque, seigneur-serf dans la société féodale ou dominant-dominé dans la société capitaliste, le rapport reste le même : quelques-uns seulement parmi les hommes ont pouvoir sur la masse. La société postindustrielle n'échappe pas à la règle. Elle réitère le même modèle de rapports sociaux sous la forme de la domination d'une élite d'intellectuels sur une masse anonyme. 


\title{
LE RAPPORT MASSE-ÉLITE COMME MODÈLE CANONIQUE DE LA DIALECTIQUE SOCIALE
}

\author{
par Christiane Gohier
}

\begin{abstract}
RÉSUMÉ. L'étude des rapports sociaux entre les hommes permet de dégager un invariant structurel des sociétés avec accumulation : le fait de la domination économico-socio-politique d'un petit nombre parmi les hommes sur le plus grand nombre. Qu'il s'agisse du couple maître-esclave dans la société grecque, seigneurserf dans la société féodale ou dominant-dominé dans la société capitaliste, le rapport reste le même : quelques-uns seulement parmi les hommes ont pouvoir sur la masse. La société postindustrielle n'échappe pas à la règle. Elle réitère le même modèle de rapports sociaux sous la forme de la domination d'une élite d'intellectuels sur une masse anonyme.

ABSTRACT. The fact of an economic, social and political domination of a minority over the majority is an invariant of all societies of accumulation in the history of mankind. Wether it be the master and slave couple in the Greek City, the Lord and Serf duality in the Feudal society or the "dominant-dominé" division of the capitalist society, the relationship is built on the same pattern : only a few men have power over the mass. The postindustrial or "programmed" society duplicates the same pattern of the domination of an Elite of Intellectuals over an anonymous mass.
\end{abstract}

Notre analyse du rapport Masse-Élite s'inscrit dans le cadre d'une recherche sur les théories de l'égalité. Elle a pour objet spécifique l'étude des rapports sociaux entre les hommes et pour paramètre, le concept d'égalité sociale.

I- QUESTION DE MÉTHODE OU MISE EN PLACE DES PRÉSUPPOSÉS THÉORIQUES QUI SOUS-TENDENT L'ANALYSE.

Pour définir et mesurer l'égalité sociale, nous nous servirons de la règle de distribution égalitarienne de F.E. Oppenheim. Le choix de cette règle repose sur sa valeur fonctionnelle en tant 
que règle-paramètre qui se veut descriptive plutôt que normative ${ }^{1}$, à savoir :

With respect to a given initial distribution, a rule of redistribution is then more egalitarian, the smaller the difference between the percentage holdings at the end in comparison with the difference at the start. ${ }^{2}$

En fonction de cette règle de distribution, la définition de l'égalitarisme retenue par Oppenheim sera le « partage équitable des biens de la communauté entre le plus grand nombre " ("Equal shares to a relatively large group" ${ }^{\text {) }}$. Ce partage suppose une redistribution économique et, conséquemment, l'égalité par rapport au statut, aux privilèges et aux avantages sociaux qui en découlent.

\section{II- QUESTION : LES RAPPORTS SOCIAUX ENTRE LES HOMMES SONT- ILS ÉGALITAIRES?}

\section{Thèse : Pour une théorie de l'inégalité}

Disons, hypothèse : Les structures politico-économiques des sociétés avec État engendrent invariablement des rapports sociaux inégalitaires entre les hommes.

Le champ d'applicabilité de la théorie de l'inégalité sociale se limite aux sociétés avec État, c'est-à-dire où la structure économique est celle de l'accumulation et le pouvoir organisé politiquement et économiquement. Les sociétés dites primitives, à économie collective - sans accumulation - sont donc exclues du champ d'applicabilité de la théorie car la redistribution des biens et le partage des tâches y sont plus égalitaires.

Reprenant à son compte les travaux ethnologiques de Pierre Clastres, François Châtelet montre bien que ce type de société n'engendre pas le rapport de domination des sociétés avec État.

1. Nous ne reprendrons pas ici tout le débat entourant la question du descriptif et du normatif. Partant du principe que nous ne nous situons jamais dans le descriptif pur, la règle de Oppenheim est dite descriptive au sens où elle pose une mesure descriptive qui va dans le sens de la prise en considération numérique des acteurs sociaux (le plus grand nombre), par opposition à un jugement de valeur purement normatif, de type méritocratique, par exemple.

2. Felix E. Oppenheim, "Egalitarianism as a descriptive concept", American Philosophtcal Quarterly, Volume 7, Number 2, April 1970, pp. 143 à 152, page 150.

3. Ibid., page 149 . 
Le chef, bien qu'il chapeaute l'organisation sociale, se voit doté d'un statut très particulier :

Celle-ci [la société primitive] sans État implique en premier lieu un statut de la chefferie qui est, par essence, différent de ce que nous appelons pouvoir ou domination politique. Car il y a un chef, mais il ne préfigure en aucune manière les despotes. Il intervient pour réduire les conflits entre individus ou entre parentés ; et sa parole dit le consensus. Cependant son intervention n'est pas pouvoir au sens où y serait incluse une force de coercition ; sa parole n'est pas celle de la loi (. . .). Bref, il est au service de la communauté. ${ }^{4}$

Dans le cas des sociétés avec État, où il y a structure d'accumulation, il y a combat pour l'accumulation et la classe sociale qui s'approprie le surproduit social tend à assurer la conservation et la défense de ses intérêts en consolidant politiquement son pouvoir économique.

Nous entendons par " classe sociale ", un groupe d'individus dans une société " $\mathrm{x}$ » ayant un vécu économique et culturel tel qu'il découpe un réseau de valeurs " y » dans un champ culturel « z": son idéologie. Chaque classe sociale produit son idéologie comme si elle définissait l'ensemble des représentations possibles dans le champ culturel (z) à une époque donnée, alors qu'elle n'en découpe qu'une partie (y). En fait, apparaît ici la question - déjà inscrite chez Marx - de la prétention de l'idéologie dominante à la représentation universelle. Nous étendons simplement le champ d'applicabilité du concept de la fausse représentation à toute idéologie. ${ }^{5}$

L'appropriation de l'accumulation par une classe sociale donnée explique l'instauration des rapports inégalitaires entre les hommes. La classe sociale au pouvoir tend en effet à défendre ses intérêts plutôt que les intérêts de la majorité et assure le maintien de son pouvoir par une élite dirigeante, élite qui est elle-même une caste à l'intérieur d'une élite qui est une classe. Cette élite dirigeante se voit gratifiée des privilèges économiques qui découlent de la possession du surproduit social et des pri-

4. Les Idéologies, sous la direction de François Châtelet et Gérard Mairet, Tome I : Des Pharaons à Charlemagne, Marabout Université, 1981 ; F. Châtelet, page 21.

5. Sur toute cette question de l'idéologie, voir Alain Touraine, Production de la société, Seuil, Paris 1973, plus spécifiquement le chapitre I portant sur " l'historicité ". 
vilèges sociaux qui découlent de sa conservation au niveau politique. Conséquence : L'exercice de la domination sociale se fait toujours par le petit nombre sur le plus grand nombre.

\section{III- VÉRIFICATION HISTORIQUE DE L'HYPOTHÈSE.}

" La dialectique historique est la dialectique du Maître et de l'Esclave " (Alexandre Kojeve commentant Hegel $)^{6}$.

Les couples paradigmatiques Maitre-Esclave, Seigneur-Serf, Dominant-Dominé, Masse-Élite sont les témoins linguistiques d'une constante dans la dialectique historique, à savoir : la domination de l'homme par l'homme, plus spécifiquement celle du pouvoir qu'exerce le petit nombre sur le plus grand.

De la cité grecque à la société capitaliste monopolistique en passant par la société féodale, le modèle se perpétue. Il s'agit de poser les couples antagonistes pour le voir.

\section{Société}

Cité grecque

Société féodale

Société capitaliste

Capitalisme monopolistique et socialisme

\section{Dominant}

Maître (homme libre)

Seigneur (maître, possesseur d'un fief)

Dominant (maître, exploiteur)

Élite (celui qui est choisi, qui se distingue du commun ; exception)

\section{Dominé}

Esclave (asservi, dont la vie a été conservée).

Serf (de "servus", esclave, dépendant du maître).

Dominé (maîtrisé, exploité).

Masse (peuple, commun ; majorité).

\section{Le tableau se termine sur les sociétés techno-bureaucratiques}

6. Alexandre Kojeve, Introduction à la lecture de Hegel, Legons sur la phénoménologie de l'esprit, N.R.F., Gallimard, 1947, page 16. En fait, Kojeve commente assez librement le texte de Hegel ici. Dans La Phénoménologie de l'esprit, traduction par Jean Hyppolite (Aubier, Montaigne) ; nous trouvons posée la dialectique du Maître et de l'Esclave comme moment de la reconnaissance de soi par l'autre, cet autre à supprimer tout en le conservant pour atteindre dans un dernier moment la conscience de soi authentique : " Ils se reconnaissent comme se reconnaissant réciproquement " (p. 157). L'esclave y parvient par son travail, par la formation de l'objet (indépendant), "la conscience travaillante en vient ainsi à l'intuition de l'être indépendant comme intuition de soi-même "(p. 165). Et c'est ici que l'être-pour-soi se réintègre vraiment « dans la formation l'être-pour-soi devient son propre être pour elle, et elle parvient à la conscience d'être elle-même en soi et pour soi »(p. 165). En ce sens on pourrait dire que c'est l'esclave qui fait réellement advenir l'être-pour-soi . . . C'est en ce sens qu'on peut dire que Hegel pose la première figure de la dialectique historique du Maître et de l'Esclave comme pôles irréductibles mais nécessaires de la dialectique sociale. 
capitalistes monopolistiques et socialistes telles que nous les connaissons. Elles sont de plus en plus dirigées par " l'intelligentsia » : élite intellectuelle possédant le monopole du savoir, du capital culturel.

Cette " élite monopolisatrice » se retrouve dans les autres types de société. Dans tous les cas, une partie des hommes (le plus grand nombre) n'a pas accès au lieu gardé du cumul (des privilèges, des richesses, du savoir) qui fonde le pouvoir économique et politique.

L'esclave est aliéné de sa liberté (statut d'homme libre); le travailleur, du produit de son travail et la masse, du haut savoir.

Si nous appliquons la règle de distribution égalitarienne de Oppenheim à ces divers types de sociétés (avec État), nous constatons que celles-ci sont inégalitaires. Au lieu de réduire les écarts économiques et sociaux entre les différentes classes sociales, comme le veut la règle de Oppenheim, les sociétés avec État tendent au contraire à justifier et à légitimer les écarts sociaux réels par le discours de l'idéologie dominante qui revêt le masque - théâtre de la fausse représentation — de l'universalité.

Le rapport inégalitaire est ainsi fondé et maintenu par les mécanismes intrinsèques à l'exercice même du pouvoir et à sa conservation : défense des intérêts d'un petit nombre qui tend à reproduire les conditions qui permettent l'exercice de sa domination.

Au lieu du partage équitable entre le plus grand nombre, on assiste à la domination économique et sociale du petit nombre sur le plus grand nombre soit, le rapport inégalitaire comme constante de la dialectique sociale dans les sociétés avec État ou

IV- LE RAPPORT MASSE-ÉLITE COMME MODÈLE CANONIQUE DE LA DIALECTIQUE SOCIALE.

Nous avons choisi le couple paradigmatique " Masse-Élite » pour désigner le rapport invariant de domination sociale parce qu'il souligne bien, croyons-nous, l'aspect privilégié du dominant (élite) et le fait de la domination du plus petit nombre sur 
le plus grand (masse). De plus, c'est celui qui, selon nous, désigne le mieux les rapports de domination qui tendent à s'instaurer dans la société contemporaine - ce que nous verrons un peu plus loin. Reste à voir comment, avec ce rapport de domination, penser la dialectique sociale ou comment, dans les sociétés avec État, le rapport de domination Masse-Élite est un rapport dialectique nécessaire à la transformation de la société.

C'est avec Hegel qu'apparaît la première figure de la dialectique historique du Maître et de l'Esclave, à partir de leur rapport réciproque et nécessaire. Marx, pour sa part, reprend le concept de dialectique dans une conception matérialiste de l'histoire où l'esclave (le prolétariat) devient la figure messianique, représentant les intérêts universels, qui supprimera le maître (la bourgeoisie) et instaurera la société égalitaire, sans classe.

Après Marx, la vision historiciste des orientations téléologiques de l'humanité s'estompe.

Nietzsche, bien qu'il annonce le Surhomme, thématise la volonté de puissance aussi bien pour l'opprimé que pour l'oppresseur :

S'il s'avérait que dans cette "volonté de morale " même ne se camoufle que cette "volonté de puissance-ci », que haine et mépris ne sont aussi qu'un vouloir de puissance, l'opprimé s'apercevrait qu'il se tient sur le même terrain que l'oppresseur et qu'il n'a aucun privilège, aucune position supérieure devant lui. ${ }^{7}$

Il annonce ainsi la perspective contemporaine de la dialectique des rapports sociaux entre le dominant et le dominé où, sous certaines conditions, l'un se transforme en l'autre sans que jamais disparaisse le rapport de domination.

Telle est la conception de la dialectique sociale thématisée par plusieurs sociologues contemporains, notamment par A. Touraine et A.W. Gouldner.

Vision pessimiste des rapports sociaux, dira-t-on ? Oui et non puisque, selon Touraine, cette dialectique assure la transformation continuelle de la société. Par une stratégie d'opposition constante au pouvoir en place (quel qu'il soit), par les

7. Nietzsche, F., Fragments Posthumes, «Le Nihilisme Européen ", Juin 1887, VIII" 5(71)9. 
changements de classe (idéologie) au pouvoir et surtout par les mouvements sociaux de contestation dans la société civile (groupuscules de gauche, écologistes ....) dont l'enjeu n'est pas le pouvoir, on empêche la « rigidification " des structures de la classe au pouvoir, partant, son hégémonie, sa tendance au fascisme et au totalitarisme. On assure également, par le fait même, une transformation constante de la société.

Gouldner dira en substance la même chose en parlant de la Nouvelle Classe au pouvoir ou plutôt de celle qui tend à prendre le pouvoir, soit la nouvelle intelligentsia composés d'intellectuels et de technologues. Cette nouvelle classe représente, sous certains aspects, des intérêts universels . . . du moins jusqu'à ce qu'elle accède définitivement au pouvoir et cherche à le consolider. C'est ce que nous verrons maintenant.

V- VÉRIFICATION DE L'HYPOTHÈSE DANS L'ANALYSSE DES RAPPORTS SOCIAUX INSTITUÉS DANS LA SOCIÉTÉ CONTEMPORAINE.

A) Retrouvons-nous le rapport Masse-Élite dans la société contemporaine ? Quelle forme prend-il ?

Pour A.W. Gouldner, il y a une montée de la classe des intellectuels comme nouvelle classe au pouvoir. ${ }^{8}$ Cette nouvelle classe est composée d'une intelligentsia technologique ("technical") et des intellectuels humanistes (Intellectuels critiques, qui ont des visées émancipatrices et décident du projet téléologique de la société).

Gouldner, d'accord avec Lourau, pose deux jalons historiques marquant l'apparition de la notion d'intellectuels :

The very term "intelligenty" was used in Russia during the 1860 s to refer to a self-conscious elite of the well educated characterized by critical tendencies toward the status quo ; the term "intellectuals" came into vogue through the Manifesto of Intellectuals protesting the French government's persecution of Dreyfuss. ${ }^{9}$

8. Nous assistons depuis quelques années à une prolifération d'ouvrages concernant l'intellectuel et le pouvoir. Nous pensons plus particulièrement ici aux ouvrages de G. Konrad et I. Szelenyi, La marche au pouvoir des intellectuels (Paris, Seuil, 1979), de René Lourau, Le lapsus des intellectuels (Paris, "Réflexion faite », Privat, 1981) et de Regis Debray, Le pouvoir intellectuel en France (Paris, Ramsay, 1980).

9. Gouldner, A.W., "The New Class Project II", Theory and society, vol. 6, no. 3, november 1978 , p. 343 à 389 , page 354 . 
La formation des intellectuels comme classe sociale ne s'explique cependant que par la structure politico-économique d'une société techno-bureaucratique où la thésaurisation du savoir comme capital culturel permet l'accession au pouvoir par la possession ou encore le contrôle des moyens de production. Les intellectuels utilisent leur savoir comme valeur d'échange monnayable en tant que force productive d'une société technologisée et informatisée.

Dans la société techno-bureaucratique capitaliste monopolistique que nous connaissons, l'intellectuel détient le pouvoir politique (représentation au sein du gouvernement et influence sur l'idéologie des partis politiques); l'intellectuel gère également de plus en plus l'économie en utilisant son pouvoir de décisions dans les grandes entreprises.

Dans la société techno-bureaucratique socialiste des pays de l'est, l'intellectuel possède à la fois le pouvoir politique et économique. L'État, par l'intermédiaire du parti, décide des orientations téléologiques de la société et de la redistribution des biens.

Pour Gouldner, la croissance de l'État est profitable aux intellectuels, car c'est à travers lui que leur savoir peut prendre forme de pouvoir.

B) Cette nouvelle classe reproduit-elle le rapport de domination Masse-Élite dans son sens fort ou est-elle plus universelle ? Représente-t-elle les intérêts de la " masse "?

Gouldner nous dit que cette "New Class" est une classe universelle imparfaite ("flawed").

UNIVERSELLE parce que

1. Elle est internationale. C'est la plus cosmopolite des élites. Son langage est international : C'est le "CCD" (critical and careful discourse) que tout intellectuel peut comprendre.

2. Elle prétend que son idéologie est neutre, non partisane (ce qui est le propre de toute légitimation politique) : une idéologie de la technologie et de la science, " apolitique ». 
The new ideology holds that productivity depends primarily on science and technology and that the society problems are solvable on a technological basis and with the use of educationally acquired technical competence. ${ }^{10}$

3. L'accès au pouvoir ne dépend pas de la richesse ou du statut social de la personne. En principe, le savoir est accessible à tout le monde.

4. Comme son pouvoir réside dans le savoir, la communication (écrite ou orale) est son bastion ; son intérêt est donc de contrer toute censure, ce qui représente un intérêt émancipateur pour la masse également en rendant possible l'exercice public de la raison critique.

5. Ses intérêts vont plus dans le sens d'améliorer ses conditions de travail que d'obtenir une rémunération plus élevée (réduction du temps de travail, par exemple) ; elle préfigure ainsi la cessation du travail aliéné. En plus, elle porte attention à l'environnement au sens large du terme - souci écologique - ce qui profite également à tous. Mais, en même temps, elle est ÉLITISTE puisque :

1. Le capital culturel du "CCD" est réservé seulement à quelquesuns. Les intellectuels possèdent un savoir quantitativement et qualitativement plus grand que celui de la «masse".

2. Les intellectuels ne valorisent que leur propre culture et non celle du simple ouvrier.

3. La réduction du temps de travail et des temps contraints est une auto-réduction du temps de travail qui ne concerne que la classe des intellectuels.

4. Le cosmopolitisme des intellectuels signifie-t-il une représentation des intérêts universels ? Pourquoi ne serait-il pas plutôt la menace d'une coalition inter-nationale de la classe des intellectuels et l'indice d'un pouvoir monopolistique mondial dont la force et l'homogénéité seraient encore plus à craindre pour la Masse (Exemple : la commission trilatérale . . .) ?

10. Gouldner, A.W., "The New Classe Project I", Theory and society, vol. 6, no. 2, september 1978, p. 153 à 203, page 174 . 
En fait, la nouvelle classe est une bourgeoisie culturelle. "The New Class (. . .) appropriates privately the advantages of an historically and collectively produced cultural capital" 11 .

Reste à répondre à la question de savoir si cette élite intellectuelle entretient des rapports plus égalitaires avec la masse que dans le cas des rapports de domination les plus forts (MaîtreEsclave, par exemple).

Prenons à nouveau la règle de distribution égalitarienne de F.E. Oppenheim, soit : "With respect to a given initial distribution, a rule of redistribution is then more egalitarian, the smaller the difference between the percentage holdings at the end in comparison with the difference at the start".

En fonction de cette règle, la nouvelle classe est plus égalitaire en ce qui concerne tout ce qui se rapporte à la "oldmoneyed class" : quand il s'agit d'enlever ou de réduire les revenus ou privilèges de celle-ci. Mais elle est anti-égalitarienne au sens où elle demande des avantages de guilde spéciaux (le pouvoir politique et des revenus particuliers) sur la base de la possession du capital culturel qu'elle ne redistribue pas également à tous (entre en jeu ici toute la problématique de la démocratisation du savoir).

Les intellectuels reproduisent alors ce que René Lourau nomme la " disjonction de Lénine », c'est-à-dire la distinction entre le socialisme et le mouvement ouvrier, l'intellectuel ayant le privilège de la conscience révolutionnaire et le rôle d'éducateur par rapport au prolétariat. L'intellectuel va chercher ses appuis dans la masse mais tend à défendre ses seuls intérêts lorsqu'il accède au pouvoir.

La nouvelle intelligentsia réitère donc le modèle MasseÉlite dans son rapport au pouvoir dans une société où l'espace public est de plus en plus déserté par une Foule Anonyme, apolitique et informe : la Masse.

Pour Gouldner, cependant, l'ascension au pouvoir de la classe des intellectuels représente un moindre mal pour la masse, car celle-ci tend et tendra à représenter les intérêts de la majorité

11. Ibid., page 169 . 
jusqu'à ce qu'elle puisse consolider son pouvoir. Or, cette ascension est longue : elle se modèle sur la révolution bourgeoise :

It will have hundred of years of development, will consolidate itself through a Reformation, and have the time thoroughly to establish its own characteristic modes of production before they cap their rise with all the trappings of political authority. ${ }^{12}$

\section{VI- EN GUISE DE CONCLUSION}

La société contemporaine, dans les deux grandes figures démocratiques (de type libéral et socialiste) qui la définissent réitère le modèle de domination Masse-Élite.

Selon la règle de redistribution de Oppenheim, la démocratie de type libéral est égalitaire, mais en partie seulement. Face aux inégalités sociales de départ, on assure aux démunis la satisfaction de besoins essentiels : il y a donc une redistribution plus égalitaire des biens. Cependant, dans un troisième temps, entre en jeu un concept de redistribution inégalitaire, normatif plutôt que descriptif, à savoir la redistribution «à chacun selon son mérite " ou la redistribution de type méritocratique.

En outre, en devenant de plus en plus monopolistique, le capitalisme tend à accroître l'écart entre les différentes classes sociales. Enfin, les intellectuels en tant que "New Class" - si cette hypothèse est avérée — réitèrent aussi le rapport de domination Masse-Élite.

Quant à la démocratie de type socialiste, elle est aussi en partie égalitaire et en partie inégalitaire. Il y a redistribution des biens, mais pas nécessairement dans un partage équitable entre tous, comme le veut la théorie et, surtout, ce pouvoir de redistribution appartient à une classe sociale, celle formée par les intellectuels du parti, qui bénéficient d'avantages sociaux inhérents au pouvoir de décision. Ces intellectuels réitèrent eux aussi le modèle de domination du petit nombre sur le plus grand nombre.

En résumé, deux constatations ressortent de notre analyse, à savoir que : Les systèmes de type démocratique ne sont pas

12. Ibid., page 180 . 
nécessairement égalitaires et que le modèle contemporain des rapports sociaux Masse (Peuple) - Élite (Intellectuels) reproduit le modèle de domination du plus petit nombre sur le plus grand.

Pour conclure, disons que la dialectique du Maître et de l'Esclave est une dialectique historique dont les deux pôles irréductibles sont nécessaires à l'interaction sociétale et à la transformation de la société. Mais cette dialectique sous-tend un modèle de domination Masse-Élite qui reproduit invariablement un rapport inégalitaire entre les hommes, ce, dans toutes les sociétés avec État.

The Communist Manifesto had held that the history of all hitherto existing society was the history of class struggles : freeman and slave, patrician and plebian, lord and serf, guildmaster and journeyman, and, then, bourgeoisie and proletariat. In this series, however, there was one unspoken regularity : the slaves did not succeed the masters, the plebians did not vanquish the patricians, the serfs did not overthrow the lords, and the journeymen did not triumph over the guildmasters. The lowliest class never came to power. Nor does it seem likely to now. ${ }^{13}$

Pour une théorie de l'inégalité . . .

Département de philosophie

Université de Montréal

13. Gouldner, A.W., "The New Class Project II", op. cit., page 386. 